\title{
EDITORIAL
}

\section{Leishmaniasis: current challenges and prospects for elimination with special focus on the South Asian region}

\author{
NADIRA D. KARUNAWEERA ${ }^{1}$ and MARCELO U. FERREIRA ${ }^{2}$ \\ ${ }^{1}$ Department of Parasitology, Faculty of Medicine, University of Colombo, Sri Lanka \\ ${ }^{2}$ Department of Parasitology, Institute of Biomedical Sciences, University of São Paulo, Brazil
}

(Received 28 February 2018; accepted 1 March 2018; first published online 12 April 2018)

\begin{abstract}
SUMMARY
Leishmania donovani, the most virulent species of Leishmania, is found in the South Asian region that harbours the majority of visceral leishmaniasis (VL) cases in the world. The traditionally accepted relationships between the causative species of Leishmania and the resultant disease phenotype have been challenged during recent years and have underscored the importance of revisiting the previously established taxonomy with revisions to its classification. The weak voice of the afflicted with decades of neglect by scientists and policy makers have led to the miserably inadequate and slow advancements in product development in the fields of diagnostics, chemotherapeutics and vector control that continue to hinder the effective management and control of this infection. Limitations notwithstanding, the regional drive for the elimination of VL initiated over a decade ago that focused on India, Nepal and Bangladesh, the three main afflicted countries in the Indian subcontinent is therefore, commendable, with the subsequent status reviews and restructuring of strategies possibly even more so. However, the renewed efforts would need to be combined with plans to combat new challenges in the South-Asian region that includes the emergence of atypical parasite variants, in order to realistically achieve the set goal of regional elimination of VL.
\end{abstract}

Key words: Neglected tropical disease, kala azar, Indian subcontinent, skin lesions, Leishmania.

\section{INTRODUCTION}

Leishmaniases are a group of parasitic diseases endemic in 98 countries worldwide, with over 350 million people living at risk and the annual case incidence ranging from 0.7 to 1.3 million (Alvar et al. 2012). The disease is prevalent both in the 'New' (South and the Central America) and the 'Old' World (Southern Europe, Africa, Middle East, Central Asia and Indian subcontinent). Of the 16 categories of neglected tropical diseases (NTDs) assessed for the period from 2005 to 2013, leishmaniasis ranks next only to malaria as the second worst in the age-standardized disabilityadjusted life years (DALYs) and second only to dengue fever in the rate of DALY increase, from 5.7 to 5.9 million (Murray et al. 2015). The disease often reaches epidemic proportions in areas of low endemicity due to natural or man-made disasters, including famine, drought, flood, earthquakes and civil wars (Reithinger and Dujardin, 2007; Bern et al. 2008). The poor socio-political background of the afflicted has largely contributed to the minimal interest shown towards leishmaniases by the policy makers, and even scientists, with resultant lack of good diagnostics and chemotherapeutic

* Corresponding author: Department of Parasitology, Faculty of Medicine, University of Colombo, Sri Lanka.

E-mail: nadira@parasit.cmb.ac.1k agents to enable effective management and control of this infection. Its apparent overlap with the spread of AIDS has highlighted the increasing threat of HIV-Leishmania co-infections, particularly in India and East Africa (Cruz et al.2006; Bern et al. 2008).

\section{PHENOTYPES AND CAUSATIVE AGENTS}

Leishmaniases are considered as a disease complex rather than a single disease. Clinical manifestations occur under three main categories, i.e. cutaneous, mucocutaneous and visceral leishmaniasis (VL) (WHO, 2017). Cutaneous leishmaniasis (CL) is caused by Old World Leishmania species such as, Leishmania major/L. tropica and is marked by the appearance of varying types of skin lesions, which are often innocuous and self-healing. Mucocutaneous leishmaniasis (MCL) is caused by New World Leishmania spp. such as L. braziliensis and is a protracted disease, resulting sometimes in extensive facial disfigurement and tissue destructions in the mouth and nose. Most cases of MCL are not lifethreatening per se. However, death could occur due to complications associated with secondary infections. VL caused by L. donovani/L. infantum is far more serious. It is often fatal, if untreated and results from systemic and progressive infection of macrophages in the reticulo-endothelial systems or 
lymphoid organs, mainly in the spleen, liver and bone marrow. Clinical manifestations of VL include hepatosplenomegaly, fever, anaemia, leucopaenia, hypergammaglobulinaemia and cachexia (Dedet and Pratlong, 2003). The development of leishmaniasis follows a chronic course lasting for months and sometimes years. Interestingly, the traditionally established relationships between the causative species of Leishmania and disease phenotype have become obscure in certain instances and the leishmaniasis situation in Sri Lanka is a case in point (Karunaweera et al. 2003), with the known visceralizing species $L$. donovani resulting in essentially dermotropic disease (Fig. 1) (Kariyawasam et al. 2018). In this context, it is noteworthy that with the accumulation of information over the years has led to the taxonomy of trypanosomatids in general, and Leishmania in particular to be revised, and therefore classical classifications may soon become outdated (Esponosa et al. 2018).

There are over 20 species of Leishmania that cause disease in humans (Maroli et al. 2013; Akhoundi et al. 2016). Transmission occurs through the bite of an infected female sandfly (Phlebotomus or Lutzomyia spp), which introduces the infective stages of Leishmania to a mammalian host. Sand flies are inconspicuous, fragile and hairy winged dipterans, similar to, but smaller in size than an average sized mosquito. Most Leishmania species are considered as zoonotic parasites with humans acting as accidental hosts (Dedet and Pratlong, 2003). The causative agent of $\mathrm{VL}$ in the Indian subcontinent, $L$. donovani is however widely considered as strictly anthroponotic, although this view remains debateable (Bhattarai et al. 2010; Ready, 2014; Akter et al. 2016).

\section{CLINICAL MANAGEMENT OF LEISHMANIASIS}

Confirmation of diagnosis of leishmaniasis is made through laboratory means. Visualization of parasites in the clinical samples from symptomatic patients constitutes the time-honoured gold standard for definitive diagnosis of leishmaniasis (de Vries et al. 2015). The routine procedures for this include microscopic examinations of Giemsa-stained smears of lesion aspirates (in the case of CL and MCL; Fig. 2) or splenic or bone marrow aspirates (in the case of VL) for the presence of amastigotes and/or cultivation of the samples in suitable media for their differentiation into and/or replication as promastigotes. These century-old practices have been gradually replaced by less cumbersome and more sensitive and specific methods, i.e. serodiagnosis for the presence of Leishmania-specific antibodies or circulating antigens and by PCR amplification of Leishmania-specific DNAs (Singh and Sundar, 2015; Akhoundi et al. 2017).

The mainstay of treatment for leishmaniasis is chemotherapy, but none of the drugs in use had been specifically designed and developed for treating this disease, i.e. antimonials (meglumine antimoniate or glucantime ${ }^{\circledR}$, sodium stibogluconate or Pentostam $\left.{ }^{\circledR}\right)$, miltefosine, pentamidine, amphotericin B, ketoconazole and paromomycin (Singh et al. $2016 a$ ). The antimonials remain as the first line of treatment for VL in most endemic settings for the past so many decades, although the mode of action of these compounds remains largely unknown. As reviewed by Hendrickx et al. (2018) and also Uliana et al. (2018), the chemotherapeutic agents widely available for treatment of leishmaniasis are toxic with prolonged use resulting in significant side-effects and even death due to renal and/or cardiac complications. Better alternatives are urgently needed with drug repurposing used as a promising strategy for finding new agents for oral or topical administration with anti-Leishmania activity (Trinconi et al. 2018). Amphotericin B-liposome (AmBisome ${ }^{\circledR}$ ), a superior but expensive drug, is limited in use in endemic areas of poverty. Appearance and spread of drug resistance is also a major cause of concern, as extensively reviewed in this special issue (Sundar and Singh, 2018). Strategies for boosting hosts' immune responses to potentiate chemotherapy are considered as important future tools to meet such challenges and are reviewed by Taslimi et al. (2018) in this special issue.

Chemotherapy of CL faces the dilemma of its necessity, due to the dogma based on its tendency for self-resolution. However, treatment hastens healing, thereby minimizes the scar formation, prevents spread, progression in to more complicated disease forms, such as MCL and helps to avoid poor responsiveness of protracted disease (Marsden, 1986; Cannella et al. 2011).

Prevention of spread, though believed as a hindrance for the potential development of herd immunity, would be important from a public health standpoint at least in countries with CL due to potentially visceralizing $L$. donovani infections (Kariyawasam et al. 2018). Alternative approaches for treating CL are now available by using physical means, e.g. thermotherapy that uses radio-frequency generated heat (RFHT) that is cost-effective and safe (Refai et al. 2017). The outcome of numerous clinical trials using RFHT for the treatment of CL caused by varying Leishmania spp. has been carefully reviewed in this special issue (David, 2018).

LEISHMANIASIS IN THE INDIAN SUBCONTINENT

The Indian subcontinent accounts for nearly $70 \%$ of the world's anthroponotic VL cases, amounting to several hundred thousand annual cases (Alvar et al. 2012). VL is however substantially under-reported, with reported coverage varying not only between countries but even between districts within a given 


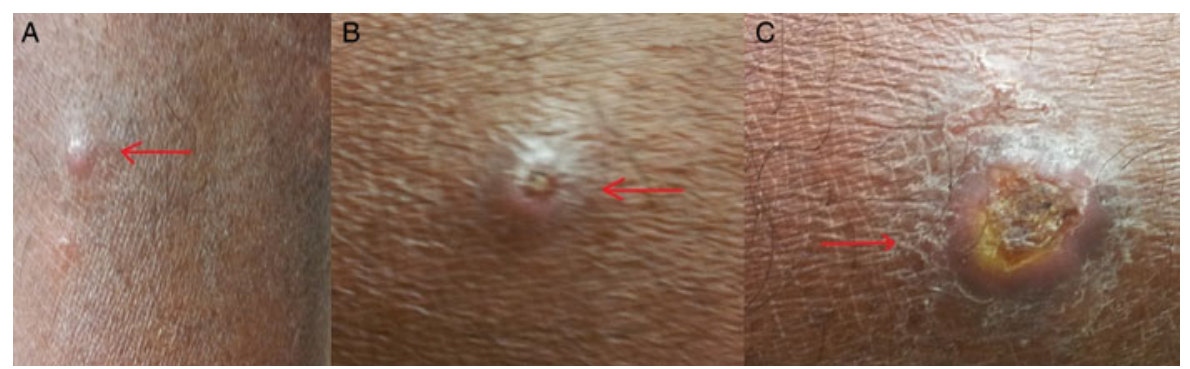

Fig. 1. Skin lesions observed in cutaneous leishmaniasis patients. (a) papule; (b) scaling nodule; (c) ulcer.

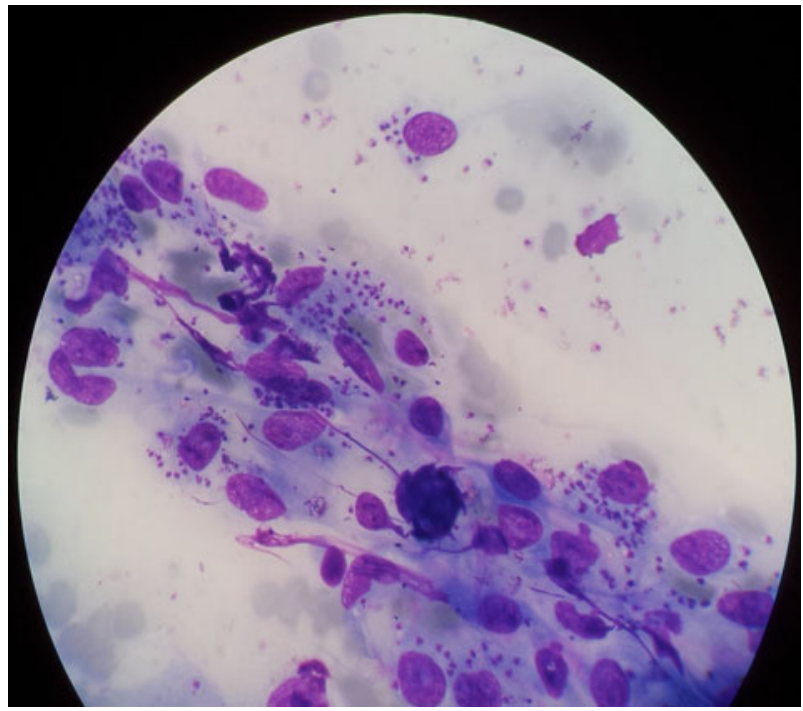

Fig. 2. Microscopic appearance of a Giemsa-stained smear made from tissue aspirates from a skin lesion of a cutaneous leishmaniasis patient [under oil immersion $(\times 1000$ magnification $)]$.

country (Bern et al. 2008; Alvar et al. 2012). India has the world's highest national VL incidence, Nepal and Bangladesh being the next. Taken together, the population at risk of acquiring $\mathrm{VL}$ is $\sim 200$ million in these three countries. VL data with regard to other south Asian countries such as Bhutan and Sri Lanka, however, are sparse with the general belief of it being sporadic and scattered upheld in most part (Alvar et al. 2012).

Clinical features of VL is marked by splenomegaly as the most noticeable symptom and is commonly accompanied by other non-specific signs such as anaemia, weight loss and hepatomegaly. Post-kala azar dermal leishmaniasis (PKDL) is also an intriguing clinical scenario described in India and occurs in 5-10\% of VL cases following apparent cure of VL (and sometimes with no history of preceding VL). PKDL patients present with macular papular skin lesions and are speculated to act as reservoirs for VL transmission (due to the parasiterich nature of the skin lesions) (Ready, 2014), although such significance of PKDL remains debatable (Le Rutte et al. 2016; Hirve et al. 2017).
Leishmaniasis is a relatively newly established disease in Sri Lanka with the first autochthonous case of CL reported in 1992 (Athukorale et al. 1992). There has been a steady increase in the numbers and distribution of CL across the country during the past two decades (http://www.epid.gov. lk/web/images/pdf/wer/2015/vol_42_no_24-english. pdf). The causative agent of CL in Sri Lanka is L. donovani, of type MON-37 (Karunaweera et al. 2003), which is indeed a cause for concern, and is transmitted by Phlebotomus argentipes, the same vector found elsewhere in the south Asian region (Gajapathy et al. 2013). Leishmania donovani is an established agent of human VL in other south Asian countries, though dermotropism, as abundantly seen in Sri Lanka, has only been occasionally observed elsewhere (Elamin et al. 2008; Kumar et al. 2015). This has led to investigations on the role for both the parasite (Siriwardana et al. 2007; Zhang et al. 2014) and host genetics in determining the disease phenotype (Samaranayake et al. 2010). However, the debate continues and the theories on CL-inducing L. donovani being 'essentially' dermotropic still remain inconclusive.

\section{ELIMINATION OF LEISHMANIASIS FROM THE INDIAN SUBCONTINENT}

A memorandum of understanding was signed in May 2005 during the World Health Assembly between the World Health Organization and the government representatives of India, Nepal and Bangladesh that committed themselves to work in mutual cooperation to achieve the elimination of VL from these countries by 2015 (World Health Organization., 2005). The objective of the VL elimination initiative as laid down at the outset was to reduce the annual incidence of $\mathrm{VL}$ below one per 10000 population at district, subdistrict or upazilla level in India, Nepal and Bangladesh, respectively (Picado et al. 2012). The strategies adopted were based on case detection and management together with vector control. Though there was a substantial progress in selected areas with conspicuous reduction of the VL burden in the region, certain gaps acted as road blocks and prevented the expected progress towards the achievement of targeted 
outcome. Such deficiencies included the low coverage of health services at community level, parasite resistance to antimonial compounds, limited availability and high cost of alternative therapeutic agents and the lack of effectiveness of vector control measures (Bhandari et al. 2011; Chowdhury et al. 2014; Muniaraj, 2014). The VL elimination framework was subsequently updated (World Health Organization, 2012a). In the new framework, there were five key strategies described, viz. early diagnosis and complete case management, effective disease and vector surveillance, social mobilization and building partnerships, and clinical and operational research, to achieve the elimination goal. A more recent initiative led the WHO to define a road map for prevention, control, elimination and eradication of 17 NTDs, including VL, as a step towards achieving the Sustainable Development Goals (World Health Organization, 2012b). These efforts included the extension of support to enable better access to drugs and related interventions, and monitor progress towards VL elimination by 2020 with all stake holder involvement (Hirve et al. 2017).

There are obvious advantages of moving towards elimination of leishmaniasis from the South Asian region. However, the deficiencies would need to be carefully addressed in order to ensure a more successful outcome at least by year 2020. In addition to the obvious factors that may have contributed to the sustained parasite transmission (Hirve et al. 2017), the presence of phenotypic/genotypic variants of $L$. donovani in the region with the potential to act as reservoirs of infection has obvious implications on the ongoing plans for the elimination of VL from the South-Asian region; a factor that has been largely overlooked thus far.

Though vector control is an essential component in the overall strategy towards disease elimination, there have been obvious lack of innovations in that field going back to many decades (Picado et al. 2012). On the other hand, there have been some notable progress in the fields of diagnostic, therapeutic and vaccine development (Singh and Sundar, 2015; Srivastava et al. 2016; Sundar and Singh, 2016), but many hurdles persist (Singh et al. 2016b). For regional elimination of L. donovani-induced leishmaniasis to become a reality, effective deployment of existing and new tools will be essential. A strong political as well as active community participation would be imperative and so would inter country cooperation and partnerships. Furthermore, appropriate diagnostic and treatment services as well as effective epidemiological surveillance also would need to be ensured in order to achieve a more successful outcome of the renewed efforts in the regional drive towards the elimination of L. donovani-induced leishmaniasis.

\section{ACKNOWLEDGEMEN'T}

Assistance provided by Ms. Udeshika Kariyawasam in formating of text and images is greatly appreciated.

\section{FINANCIAL SUPPORT}

Financial support for NDK is through the National Institute of Allergy and Infectious Diseases of the National Institutes of Health under award numbers R01AI099602 and U01AI136033. The content of this editorial is solely the responsibility of the authors and does not necessarily represent the official views of the National Institutes of Health.

\section{CONFLICT OF INTEREST}

None.

\section{REFERENCES}

Akhoundi, M., Kuhls, K., Cannet, A., Votýpka, J., Marty, P., Delaunay, P. and Sereno, D. (2016). A historical overview of the classification, evolution, and dispersion of leishmania parasites and sandflies. PLoS Neglected Tropical Diseases 10, 1-40.

Akhoundi, M., Downing, T., Votýpka, J., Kuhls, K., Lukeš, J., Cannet, A., Ravel, C., Marty, P., Delaunay, P., Kasbari, M., Granouillac, B., Gradoni, L. and Sereno, D. (2017). Leishmania infections: molecular targets and diagnosis. Molecular Aspects of Medicine 57, $1-29$.

Akter, S., Alam, M.Z., Nakao, R., Yasin, M. G., Kato, H. and Katakura, K. (2016). Molecular and serological evidence of leishmania infection in stray dogs from visceral leishmaniasis-endemic areas of Bangladesh. American Fournal of Tropical Medicine and Hygiene 95, 795-799.

Alvar, J., Vélez, I. D., Bern, C., Herrero, M., Desjeux, P., Cano, J., Jannin, J. and de Boer, M. (2012). Leishmaniasis worldwide and global estimates of its incidence. PLoS ONE 7, 1-12.

Athukorale, D. N., Seneviratne, J. K., Ihalamulla, R. L. and Premaratne, U. N. (1992). Locally acquired cutaneous leishmaniasis in Sri Lanka. The Fournal of Tropical Medicine and Hygiene 95, 432-433.

Bern, C., Maguire, J. H. and Alvar, J. (2008). Complexities of assessing the disease burden attributable to leishmaniasis. PLoS Neglected Tropical Diseases 2, e313.

Bhandari, G. P., Angdembe, M. R., Rijal, S. and Boelaert, M. (2011). Will visceral leishmaniasis be eliminated from Nepal? A review of recent (1994-2006) control efforts. Nepal Medical College Fournal: NMCF 13, 220-225.

Bhattarai, N. R., Van Der Auwera, G., Rijal, S., Picado, A., Speybroeck, N., Khanal, B., De Doncker, S., Das, M. L., Ostyn, B., Davies, C., Coosemans, M., Berkvens, D., Boelaert, M. and Dujardin, J. C. (2010). Domestic animals and epidemiology of visceral leishmaniasis, Nepal. Emerging Infectious Diseases 16, 231-237.

Cannella, A.P., Nguyen, B. M., Piggott, C.D., Lee, R.A., Vinetz, J. M. and Mehta, S. R. (2011). Short report: a cluster of cutaneous leishmaniasis associated with human smuggling. American fournal of Tropical Medicine and Hygiene 84, 847-850.

Chowdhury, R., Mondal, D., Chowdhury, V., Faria, S., Alvar, J., Nabi, S. G., Boelaert, M. and Dash, A.P. (2014). How far are we from visceral leishmaniasis elimination in Bangladesh? An assessment of epidemiological surveillance data. PLoS Neglected Tropical Diseases 8, e3020. Cruz, I., Nieto, J., Moreno, J., Cañavate, C., Desjeux, P. and Alvar, J. (2006). Leishmania/HIV co-infections in the second decade. Indian Fournal of Medical Research 123, 357-388.

David, J. R. (2018). The successful use of radiofrequency-induced heat therapy for cutaneous leishmaniasis: a review. Parasitology. This issue. doi:10.1017/S0031182018000124.

de Vries, H. J. C., Reedijk, S. H. and Schallig, H. D. F.H. (2015). Cutaneous leishmaniasis: recent developments in diagnosis and management. American Fournal of Clinical Dermatology 16, 99-109.

Dedet, J. P. and Pratlong, F. (2003). Leishmaniasis. In Cook, G. and Zumla, A. (eds). Manson's Tropical Diseases, pp. E.L.S.T. with Saunders. London: Elsevier Science, pp. 1339-1364. 
Elamin, E. M., Guizani, I., Guerbouj, S., Gramiccia, M., El Hassan, A.M., Di Muccio, T., Taha, M. A. and Mukhtar, M. M. (2008). Identification of Leishmania donovani as a cause of cutaneous leishmaniasis in Sudan. Transactions of the Royal Society of Tropical Medicine and Hygiene 102, 54-57.

Esponosa, O. A., Serrano, M. G., Camargo, E. P., Teixeira, M. M. G. and Shaw, J. J. (2018). An appraisal of the taxonomy and nomenclature of trypanosomatids presently classified as Leishmania and Endotrypanum. Parasitology. This Issue. doi:10.1017/S0031182016002092.

Gajapathy, K., Peiris, L. B. S., Goodacre, S. L., Silva, A., Jude, P. J. and Surendran, S. N. (2013). Molecular identification of potential leishmaniasis vector species within the Phlebotomus (Euphlebotomus) argentipes species complex in Sri Lanka. Parasites \& Vectors 6, 302.

Hendrickx, S., Guerin, P., Caljon, G., Croft, S. and Maes, L. (2018). Evaluating drug resistance in visceral leishmaniasis: the challenges. Parasitology. This Issue. doi:10.1017/S0031182016002031.

Hirve, S., Kroeger, A., Matlashewski, G., Mondal, D., Banjara, M. R., Das, P., Be-Nazir, A., Arana, B. and Olliaro, P. (2017). Towards elimination of visceral leishmaniasis in the Indian subcontinent - translating research to practice to public health. PLoS Neglected Tropical Diseases 11, 1-25.

Kariyawasam, K., Selvapandiyan, A., Siriwardana, H., Dube, A., Karunanayake, P., Senanayake, S., Dey, R., Gannavaram, S., Nakhasi, H. and Karunaweera, N. (2018). Dermotropic Leishmania donovani in Sri Lanka: visceralizing potential in clinical and preclinical studies. Parasitology, This Issue. doi:10.1017/S003118201700169X.

Karunaweera, N. D., Pratlong, F., Siriwardane, H. V.Y. D., Ihalamulla, R. L. and Dedet, J. P. (2003). Sri Lankan cutaneous leishmaniasis is caused by Leishmania donovani zymodeme MON-37. Transactions of the Royal Society of Tropical Medicine and Hygiene 97, 380-381

Kumar, N.P., Srinivasan, R., Anish, T.S., Nandakumar, G. and Jambulingam, P. (2015). Cutaneous leishmaniasis caused by Leishmania donovani in the tribal population of the Agasthyamala biosphere reserve forest, Western Ghats, Kerala, India. Fournal of Medical Microbiology 64, 157-163.

Le Rutte, E. A., Coffeng, L.E., Bontje, D. M., Hasker, E. C., Ruiz Postigo, J. A., Argaw, D., Boelaert, M. C. and De Vlas, S. J. (2016). Feasibility of eliminating visceral leishmaniasis from the Indian subcontinent: explorations with a set of deterministic age-structured transmission models quantitative analysis of strategies to achieve the 2020 goals for neglected tropical diseases: where are we now? Parasites and Vectors 9, 1-14.

Maroli, M., Feliciangeli, M.D., Bichaud, L., Charrel, R. N. and Gradoni, L. (2013). Phlebotomine sandflies and the spreading of leishmaniases and other diseases of public health concern. Medical and Veterinary Entomology 27, 123-147.

Marsden, P. D. (1986). Mucosal leishmaniasis ('espundia' escomel, 1911) Transactions of the Royal Society of Tropical Medicine and Hygiene 80, 859-876. Muniaraj, M. (2014). The lost hope of elimination of kala-azar (visceral leishmaniasis) by 2010 and cyclic occurrence of its outbreak in India, blame falls on vector control practices or co-infection with human immunodeficiency virus or therapeutic modalities? Tropical Parasitology 4, 10-19.

Murray, C. J. L., Barber, R. M., Foreman, K. J., Ozgoren, A. A., AbdAllah, F., Abera, S. F., Aboyans, V., Abraham, J. P., Abubakar, I., Abu-Raddad, L. J., Abu-Rmeileh, N. M., Achoki, T., Ackerman, I. N., Ademi, Z., Adou, A. K., Adsuar, J. C., Afshin, A., Agardh, E. E., Alam, S. S., Alasfoor, D., Albittar, M. I., Alegretti, M. A., Alemu, Z. A., Alfonso-Cristancho, R., Alhabib, S., Ali, R., Alla, F., Allebeck, P., Almazroa, M. A., Alsharif, U., et al. (2015). Global, regional, and national disability-adjusted life years (DALYs) for 306 diseases and injuries and healthy life expectancy (HALE) for 188 countries, 1990-2013: quantifying the epidemiological transition. The Lancet 386, 2145-2191.
Picado, A., Dash, A. P., Bhattacharya, S. and Boelaert, M. (2012). Vector control interventions for visceral leishmaniasis elimination initiative in. Indian Fournal of Medical Research 136, 22-31.

Ready, P. (2014). Epidemiology of visceral leishmaniasis. Clinical Epidemiology 6,147.

Refai, W. F., Weerasingha, S., Sumanasena, B., Madarasingha, N. P., Karunaweera, N.D., Fernandopulle, R., De Silva, A. and Satoskar, A. R. (2017). Efficacy, safety and cost-effectiveness of thermotherapy in the treatment of Leishmania donovani-induced cutaneous leishmaniasis: a randomized controlled clinical trial. American fournal of Tropical Medicine and Hygiene 97, 1120-1126.

Reithinger, R. and Dujardin, J. C. (2007). Molecular diagnosis of leishmaniasis: current status and future applications. Fournal of Clinical Microbiology 45, 21-25.

Samaranayake, T. N., Fernando, S.D. and Dissanayake, V.H.W. (2010). Candidate gene study of susceptibility to cutaneous leishmaniasis in Sri Lanka. Tropical Medicine \& International Health: TM \& IH 15, 632-638. Singh, O.P. and Sundar, S. (2015). Developments in diagnosis of visceral leishmaniasis in the elimination era. Fournal of Parasitology Research, 1-10. doi:10.1155/2015/239469.

Singh, O. P., Singh, B., Chakravarty, J. and Sundar, S. (2016a). Current challenges in treatment options for visceral leishmaniasis in India: a public health perspective. Infectious Diseases of Poverty 5, 1-15.

Singh, O.P., Hasker, E., Boelaert, M. and Sundar, S. (2016b) Elimination of visceral leishmaniasis on the Indian subcontinent. The Lancet Infectious Diseases 16, e304-e309.

Siriwardana, H., Noyes, M., Beeching, N. J., Chance, M. L., Karunaweera, N. D. and Bates, P. A. (2007). Leishmnania dinovan and cutaneous leishmaniasis, Sri Lanka. Emerging infectious diseases 13, $1-3$

Srivastava, S., Shankar, P., Mishra, J. and Singh, S. (2016) Possibilities and challenges for developing a successful vaccine for leishmaniasis. Parasites and Vectors 9, 1-15.

Sundar, S. and Singh, A. (2016). Recent developments and future prospects in the treatment of visceral leishmaniasis. Therapeutic Advances in Infectious Disease 3, 98-109.

Sundar, S. and Singh, A. (2018). Chemotherapeutics of visceral leishmaniasis: present and future developments. Parasitology. This Issue doi:10.1017/S0031182017002116.

Taslimi, Y., Zahedifard, F. and Rafati, S. (2018). Leishmaniasis and various immunotherapeutic approaches. Parasitology. This Issue. doi:10.1017/S003118201600216X

Trinconi, C. T., Reimao, J. Q., Bonano, V. I., Espada, C. R., Miguel, D. C., Yokoyama-Yasunaka, U. J. K. and Uliana, S. R. B. (2018). Topical tamoxifen in the therapy of cutaneous leishmaniasis. Parasitology. This Issue. doi:10.1017/S0031182017000130.

Uliana, S. R. B., Trinconi, C. T. and Coelho, A. C. (2018). Chemotherapy of leishmaniasis: present challenges. Parasitology. This Issue. doi:10.1017/ S0031182016002523.

WHO (2017). Leishmaniasis. WHO fact sheet. World Health Organization, Geneva, Switzerland.

World Health Organization (2005). Regional Strategic Framework for Elimination of Kala-Azar From the South-East Asia Region (2005-2015). Regional Office for South-East Asia SEA-VBC-85 (Rev-1), New Delhi.

World Health Organization (2012a). Regional Strategic Framework for Elimination of Kala-azar from the South-East Asia 2011-2015. Regional office for South-East Asia, New Delhi, India.

World Health Organization (2012b). Accelerating Work to Overcome the Global Impact of Neglected Tropical Diseases: A Roadmap for Implementation. World Health Organization, Geneva, Switzerland, pp. 1-42.

Zhang, W. W., Ramasamy, G., McCall, L. I., Haydock, A., Ranasinghe, S., Abeygunasekara, P., Sirimanna, G., Wickremasinghe, R., Myler, P. and Matlashewski, G. (2014). Genetic analysis of Leishmania donovani tropism using a naturally attenuated cutaneous strain. PLoS Pathogens 10, e1004244. 